\title{
Biología y pesquería de Panulirus gracilis (Streets 1871) (Decapoda: Palinuridae) en el Pacífico Occidental de Panamá
}

\author{
Biology and fishery of Panulirus gracilis (Streets 1871) \\ (Decapoda: Palinuridae) in the Panama's Western Pacific
}

Ángel J. Vega ${ }^{*}$, Yolani A. Robles P. ${ }^{1}$ y David Gil

\begin{abstract}
RESUMEN
La langosta espinosa del Pacífico (Panulirus gracilis) es un importante recurso pesquero del Pacífico Occidental de Panamá. Entre octubre de 2005 y abril de 2007 la población de langosta del Golfo de Montijo se muestreó para determinar LT, LC y LA, el peso total, sexo, desarrollo gonadal y la frecuencia de hembras grávidas. Además, datos de volúmenes de desembarque procedentes del Parque Nacional Marino Golfo de Chiriquí fueron utilizados para estimar la captura por unidad de esfuerzo (CPUE) (kg de langosta desembarcada / pescador-semana) entre los años 2000 y 2009. Para el Golfo de Montijo, la LT (media \pm DE) de las langostas fue de $206 \pm 31 \mathrm{~mm}$ y las hembras resultaron más grandes y pesadas que los machos. La frecuencia acumulada de la LC y del abdomen indicó que un alto porcentaje de las capturas ocurren por debajo de la talla mínima legal cuando la regulación se aplica por la medida del abdomen $(112 \mathrm{~mm})$ y es mayor en machos que en hembras. Esta diferencia es consecuencia de una regulación que no se basó en la morfometría de P. gracilis y de la presunción de que la LA es el doble de la LC. Se observaron hembras con gónadas maduras o con huevos durante todo el periodo de muestreo, lo que indica una reproducción continua. La proporción sexual favoreció a los machos (1.31:1). Entre el 2000 y el 2009 se observó una importante reducción del volumen y de la CPUE.
\end{abstract}

Palabras claves: Langosta verde espinosa, Golfo de Montijo, islas Paridas, Panulirus gracilis, reproducción.

\begin{abstract}
The Pacific spiny lobster (Panulirus gracilis) is an important fishery resource from the Western Pacific region of Panama. Between October 2005 and April 2007, the Gulf of Montijo lobster population was sampled to determine total length (LT), cephalothorax length (LC), abdominal length (LA), total weight, sex, gonadal development, and frequency of gravid females. Furthermore, lobster landing volumes from the Gulf of Chiriquí National Marine Park were used to estimate the catch per unit effort (CPUE) (kg of lobster landed / fisherman-week) between 2000 and 2009. Lobster LT in the Gulf of Montijo (average \pm SD) was $206 \pm 31 \mathrm{~mm}$ and females were significantly larger in size and weight than males. The cumulative frequency of cephalothorax and abdominal lengths indicated that a high percentage of catches occur below the minimum legal size when regulation is applied measuring the abdomen $(112 \mathrm{~mm})$. Incidence of such cases is greater in males than in females. This difference might be a consequence of a regulation lacking morphometric data for $P$. gracilis and the assumption that the abdominal length is twice the cephalothorax length. Females with mature gonads and eggs were observed throughout the sampling period, which is indicative of continuous reproduction. Sex ratio was male biased (1.31:1). Between 2000 and 2009 there was an important reduction in volume and CPUE.
\end{abstract}

Keywords: Green spiny lobster, Gulf of Montijo, Paridas Island, Panulirus gracilis, reproduction.

1 Escuela de Biología, Universidad de Panamá. Apartado 0923-00173, Santiago-Veraguas, Panamá; angeljv@ cwpanama.net*,yolrobles@hotmail.com.

2 Programa de Maestría en Ecología de Zonas Costeras. Universidad de Panamá.

Recibido 25 VI 2012

Aceptado 16 VII 2013

DOI: http://dx.doi.org/10.15359/revmar.5.1 


\section{INTRODUCCIÓN}

La langosta verde espinosa del Pacífico, Panulirus gracilis (Streets, 1871), se distribuye desde México hasta Perú. La talla máxima registrada para la especie es de $165 \mathrm{~mm}$ de longitud del cefalotórax (LC), lo que equivale aproximadamente a $370 \mathrm{~mm}$ de longitud total (LT) (Fischer et al. 1995). Vive asociada a zonas rocosas, hasta los 22 $\mathrm{m}$ de profundidad, aunque en Costa Rica se le captura con compresor hasta 18 brazas (poco más de $30 \mathrm{~m}$ ) (Guzmán et al. 2008; Naranjo, 2010).

La reproducción de esta especie es continua, con desoves múltiples a lo largo del año, con tallas de primera madurez estimadas entre 45 y $60 \mathrm{~mm}$ de LC y propuestas de talla mínima de captura por encima de los $70 \mathrm{~mm}$ de LC (Briones et al. 1981; PérezGonzález et al. 1992; Salazar-Navarro et al. 2006; Velásquez-Chavarría \& Gutiérrez-García, 2006; Guzmán et al. 2008).

La pesca de langosta en Panamá está regulada por el Decreto Ejecutivo No. 15 del 30 de marzo de 1981. Este decreto aplica para la langosta del Caribe (P. argus) y para la especie del Pacífico ( $P$. gracilis) y establece la necesidad de un permiso de pesca de langosta para poder dedicarse a la actividad, regula la talla mínima de captura en $60 \mathrm{~mm}$ de longitud del cefalotórax o $120 \mathrm{~mm}$ de longitud de cola y establece un peso mínimo de 2 oz de cola; igualmente, prohíbe la pesca y posesión de langostas con huevos y la pesca con arpón y por buceo autónomo (con scuba). Para el Caribe, como política regional, se estableció el Reglamento OSP-02-09 para el Ordenamiento Regional de la Pesquería de la Langosta del Caribe (P. argus), que entre otras medidas establece una veda regional entre el 1 de marzo y el 30 de junio (OSPESCA, 2009).

En los pocos estudios que se han realizado en Panamá sobre langosta, se reportan bajas densidades de las especies con interés comercial $P$. argus en el Caribe y $P$. gracilis en el Pacífico (Castillo \& Lessios, 2001; Guzmán \& Tewfik, 2004; Guzmán et al. 2008).

En el Pacífico panameño, la langosta se explota a lo largo de la zona costera e islas, con trasmallos langosteros o por medio de buceo a pulmón. La fuerte presión pesquera sobre el recurso ha llevado a una disminución de las densidades, hasta valores promedios menores a seis individuos por hectárea (Guzmán et al. 2008). Estos autores recomiendan un cambio en la legislación para este recurso, estableciendo una talla mínima de captura de $84 \mathrm{~mm}$ de LC (en contraste con los $60 \mathrm{~mm}$ que contempla la legislación actual), además del establecimiento de áreas de no extracción, vedas temporales, educación sobre medidas de conservación y monitoreo constante, como medidas para asegurar la sostenibilidad del recurso.

Este trabajo describe la forma en que se realizan las actividades de pesca, la estructura de tallas, pesos y aspectos de la biología reproductiva y de la pesca artesanal de P. gracilis, en dos localidades del Pacífico Occidental de Panamá. 


\section{MATERIALES Y MÉTODOS}

Los muestreos de langosta fueron realizados en el Golfo de Montijo (GM), localizado al oriente del Golfo de Chiriquí, Panamá. El GM es un sistema estuarino, cuya área interior está bordeada por manglares y en su parte media-externa se localizan playas arenosas y costas rocosas. En el del Golfo se localizan islas habitadas (Leones, Gobernadora y
Cébaco) y otras islas y promontorios rocosos menores. El sitio forma parte del Sistema Nacional de Áreas Protegidas de la Autoridad Nacional del Ambiente (ANAM), bajo la categoría de sitio Ramsar. En su condición de sistema estuarino, presenta variaciones de la salinidad, temperatura, oxígeno disuelto y transparencia asociadas al régimen de precipitación y mareas (Cámara et al. 2004; Vega, 2004) (Cuadro 1).

Cuadro 1. Valores de variables físicas y químicas en el Golfo de Montijo. Los valores representan ámbitos anuales de variación dentro del Golfo de Montijo. Fuente: Vega, 2004

Table 1. Physical and chemical variables in the Gulf of Montijo. Values represent annual ranges of variation within the Gulf of Montijo. Source: Vega, 2004

\begin{tabular}{llccc}
\hline & $\mathbf{T}^{\circ} \mathbf{C}$ & $\mathbf{S}(\mathbf{U P S})$ & $\mathbf{O}_{2}(\mathbf{m g} / \mathbf{l})$ & Secchi $(\mathbf{c m})$ \\
\cline { 2 - 5 } Mínimo & 25.8 & 0.1 & 3.3 & 20 \\
Promedio & 28.39 & 21.13 & 4.88 & 143 \\
Máximo & 30.6 & 36.4 & 7.55 & 360 \\
\hline
\end{tabular}

Muestreos en el GM: Las langostas analizadas se obtuvieron de los desembarques de la pesca artesanal en las comunidades de Palo Seco $\left(07^{\circ} 35^{\prime} 27^{\prime}, \mathrm{N}, 80^{\circ} 58^{\prime} 41^{\prime \prime} \mathrm{W}\right)$, Torio $\left(07^{\circ} 32^{\prime} 53^{\prime \prime} \mathrm{N}, 80^{\circ} 56^{\prime} 58^{\prime \prime} \mathrm{W}\right)$ y Santa Catalina $\left(07^{\circ} 37^{\prime} 03^{\prime}\right.$ ' N, $81^{\circ} 14^{\prime}$ 18 " W). Cada localidad se visitó una vez por mes, en campañas de tres días por localidad, durante 18 meses, entre octubre de 2005 y abril de 2007. En algunas ocasiones se acompañó a los pescadores a sus faenas de pesca.

Durante los muestreos de campo se realizaron observaciones sobre la forma en que se realiza la actividad extractiva de la langosta. Estas observaciones incluían características de los botes (eslora, propulsión), cantidad de pescadores por embarcación, artes y métodos de pesca y duración de la actividad de pesca.

A las langostas capturadas se les registraron: la longitud total (LT), la longitud del cefalotórax (LC) y la longitud del abdomen (LA). Todas las medidas de longitud se tomaron con un calibrador Vernier (precisión $=0.01 \mathrm{~mm})$ y el peso total $(\mathrm{PT}) \mathrm{de}$ cada organismo (g) se registró con una balanza digital (precisión $=0.1 \mathrm{~g}$ ) .

Los aspectos reproductivos estudiados fueron: composición sexual de las capturas, presencia de huevos en los pleópodos y estadio gonádico.

El sexo se determinó con base en las características de los pleópodos, sencillos en los machos y dobles en las hembras. A cada hembra se le determinó la presencia o ausencia de 
huevos en el abdomen y se verificó la condición gonadal mediante el uso de una escala de colores, que describe cuatro estadios de desarrollo gonadal que se corresponden con cambios en el color y tamaños de las gónadas en hembras, según la descripción presentada por Buesa \& Mota-Alves (1970) y Cruz (2002). La relación de la condición de desarrollo-color es la siguiente:

Condición I: color rosado (reposo o regresión).

Condición II: inicio del desarro1lo, gónadas filamentosas de color blanco o grisáceo.

Condición III: desarrollo medio, color crema-amarillo-chocolate.

Condición IV: desarrollo final o preovulatorio, que corresponde a tonalidades de rojo que culmina con un rojo intenso.

\section{Datos procedentes del Parque Nacional Marino Golfo de Chiriquí (PNMGCH): A la comunidad de} Boca Chica $\left(08^{\circ} 12^{\prime} 03^{\prime \prime} \mathrm{N}\right.$ y $82^{\circ}$ 12 ' 03" W) llegan los pescadores a vender sus langostas cada semana de capturas provenientes de áreas cercanas a arrecifes de coral que circundan las islas Paridas $\left(08^{\circ}\right.$ 07' 39" N y $82^{\circ} 19^{\prime} 06^{\prime \prime}$ W) y otras zonas del PNMGCH. También se les captura fuera del área protegida, en zonas rocosas que bordean la costa hacia Punta Bejuco (08 9' 42.6” N y $82^{\circ} 08^{\prime} 31^{\prime}$ W). En Boca Chica, un intermediario realiza la compra, llevando un registro de captura de langosta desembarcada $(\mathrm{kg})$ por categoría de comercialización (2 a $4 \mathrm{oz} \mathrm{y} \mathrm{más} \mathrm{de} 4 \mathrm{oz}$ ) y el número de pescadores a los cuales se compró la langosta. El registro lo inició en el año 2000 y lo ha mantenido hasta el año 2009. Con esta información se estimó la captura por unidad de esfuerzo (CPUE) medida como los kilogramos de langosta desembarcada por semana entre la cantidad de pescadores que desembarcaron el producto, es decir, $\mathrm{kg} /$ pescador/semana.

A través de entrevistas personales con los pescadores se obtuvo información sobre la actividad pesquera. Estas observaciones incluían características de los botes (eslora, propulsión), cantidad de pescadores por embarcación, artes y métodos de pesca y duración de la actividad de pesca.

Para el análisis de los datos de tallas mensuales se utilizó un ANOVA de una vía, previa comprobación de la homogeneidad de varianzas con el estadístico de Levene, y para la comparación de promedios la prueba de contraste de Tukey; para la comparación de tallas y pesos entre hembras y machos, se aplicó la prueba $\mathrm{t}$ de Student, la relación entre los volúmenes y el esfuerzo se analizó a través de la Correlación de Spearman y la proporción sexual a través de una Chi cuadrada con la corrección de Yates (Zar, 1999). 
En todos los casos se consideró un $\alpha=0.05$. Los análisis estadísticos y gráficos fueron realizados con el programa SPSS 15.0 (2006).

\section{RESULTADOS}

\section{Descripción de la actividad de} pesca: La pesca artesanal de langosta en el GM y en el PNMGCH se desarrolla en los alrededores de islas, promontorios rocosos, áreas de arrecifes de coral y costa rocosa.

La actividad se realiza con embarcaciones de madera o fibra de vidrio, de entre 4.5 y $9 \mathrm{~m}$ de eslora, impulsadas por motores fuera de borda (15 a 40 HP). Cuando se utilizan trasmallos para la pesca, cada embarcación lleva un motorista y 1 o 2 marinos. Cuando es buceo, la embarcación lleva un motorista y hasta 4 buzos.

En ambas localidades,
para las capturas utilizan: a) trasmallos langosteros de hilo o de monofilamento, con apertura de malla entre $10.16 \mathrm{~cm}$ (malla de 4 in) y $17.75 \mathrm{~cm}$ (malla de $7 \mathrm{in}$ ), medidos entre nudos opuestos. Los trasmallos son colocados en horas de la mañana, alrededor de áreas rocosas y revisados cada $24 \mathrm{~h}$, para recolectar los individuos enmallados. El tiempo de permanencia de los trasmallos en el agua varió, en algunos casos hasta que el trasmallo necesitara reparación, o bien hasta que las capturas fuesen tan bajas, que no valía la pena el esfuerzo. b) buceo en apnea. En el caso del GM, se apoyan con arpones artesanales, llamados "chuzos" por los pescadores. Esta técnica, a pesar de estar prohibida, es utilizada cuando el agua presenta suficiente claridad, generalmente hacia la temporada seca (diciembreabril). Para el PNMGCH, el buceo se realiza todo el año y la captura se efectúa manualmente, sin el uso de arpones.

Las langostas después de capturadas o al llegar a puerto son descabezadas y la cola se introduce en agua dulce, lo que según los pescadores mejora la consistencia del producto. En el PNMGCH, las langostas son mantenidas vivas en jaulas de madera (8 a 10 días), hasta su traslado al punto de venta. La libra de cola se pagó al pescador, aproximadamente 9 US\$. Durante los muestreos se pudo observar a promotores turísticos del área comercializando langostas tan pequeñas, que no clasificaban para el comercio legal, posiblemente para ser ofrecidas como parte del menú a los visitantes.

Tallas y pesos de langosta para el GM: La longitud total de las langostas comercializadas osciló entre 126 y $298 \mathrm{~mm}$ (Media $=206 \pm$ 31 D.E.). La longitud del cefalotórax varió entre 45 y $124 \mathrm{~mm}$ (Media $=81 \pm 15$ D.E.) y la longitud del abdomen, entre 81 y $180 \mathrm{~mm}$ (Media $=125 \pm 19$ D.E.) (Cuadro 2). 
Cuadro 2. Morfometría de Panulirus gracilis capturadas en el Golfo de Montijo, Pacífico de Panamá, entre octubre de 2005 y abril de 2007. LT: longitud total, PT: peso total, LC: longitud del cefalotórax, LA: longitud del abdomen y $\mathrm{N}$ : número de individuos Table 2. Morphometry of Panulirus gracilis caught in the Gulf of Montijo, Pacific Panama, between October 2005 and April 2007. LT: total length, PT: total weight, LC: cephalothorax length and LA: abdominal length, and N: number of individuals

\begin{tabular}{lccccc}
\hline & Promedio & Máximo & Mínimo & Desviación estándar & N \\
\hline LT $(\mathrm{mm})$ & 206 & 298 & 126 & 31 & 691 \\
PT $(\mathrm{g})$ & 351 & 936 & 57 & 147 & 686 \\
LC $(\mathrm{mm})$ & 81 & 124 & 45 & 15 & 691 \\
LA $(\mathrm{mm})$ & 125 & 180 & 81 & 19 & 688 \\
\hline
\end{tabular}

La comparación de tallas (LT) y $\quad(\mathrm{t}=12.5, \mathrm{gl}=682, P<0.05)$ y más pesos promedios por sexo indicó que pesadas $(\mathrm{t}=8.7, \mathrm{gl}=679, P<0.05)$ las hembras fueron de mayor tamaño que los machos (Cuadro 3 ).

Cuadro 3. Morfometría según sexo para Panulirus gracilis capturadas en el Golfo de Montijo entre octubre de 2005 y abril de 2007. LT: longitud total, LC: longitud del cefalotórax, LA: longitud del abdomen, PT: peso total $(\mathrm{g})$, sexo (S) H: hembras, M: machos, $\mathrm{N}$ : número de individuos

Table 3. Morphometry according to sex for Panulirus gracilis caught in the Gulf of Montijo between October 2005 and April 2007. LT: total length, LC: cephalothorax length, LA: abdominal length, PT: total weight (g), sex (S) H: female, M: male, N: number of individuals

\begin{tabular}{lcccc}
\hline & S & N & Promedio & Desviación estándar \\
\hline \multirow{2}{*}{ LT $(\mathrm{mm})$} & H & 299 & 221.07 & 27.51 \\
& M & 392 & 194.54 & 27.60 \\
LC $(\mathrm{mm})$ & H & 299 & 84.75 & 13.69 \\
& M & 399 & 78.89 & 14.96 \\
LA $(\mathrm{mm})$ & H & 299 & 136.32 & 17.11 \\
& M $(\mathrm{g})$ & 392 & 115.44 & 15.03 \\
& H & 296 & 404.55 & 139.29 \\
\hline
\end{tabular}

La longitud total promedio mensual para las langostas capturadas mostró diferencias significativas, tanto para machos como para hembras $(P<0.05)$. Sin embargo, las diferencias entre meses no presentan un patrón, ya que para los mismos meses en diferentes años, las longitudes medias fueron diferentes. Por ejemplo, en octubre y noviembre de 2005 se encontraron las tallas promedios más pequeñas, sin que hubiera diferencias estadísticas entre ellas $(P>0.05)$ y en los mismos meses, en el 2006, se presentaron las 
tallas promedios más grandes $(P<$ 0.05) (Fig. 1).

El análisis de frecuencias relativas acumuladas de la LC para captura con trasmallo indicó que el $50 \%$ de la población quedó retenida a los 84.2 $\mathrm{mm}$ para las hembras y a los $78.2 \mathrm{~mm}$ para los machos. Si el análisis se aplica con base en la longitud abdominal (LA), el $50 \%$ de la población quedó retenida por debajo de los $115.0 \mathrm{~mm}$ para los machos y de los $135.8 \mathrm{~mm}$ para las hembras. Al comparar con la talla mínima legal $(\mathrm{LC}=60 \mathrm{~mm})$, el $13.7 \%$ de las capturas estuvieron por debajo para los machos y el 3\%

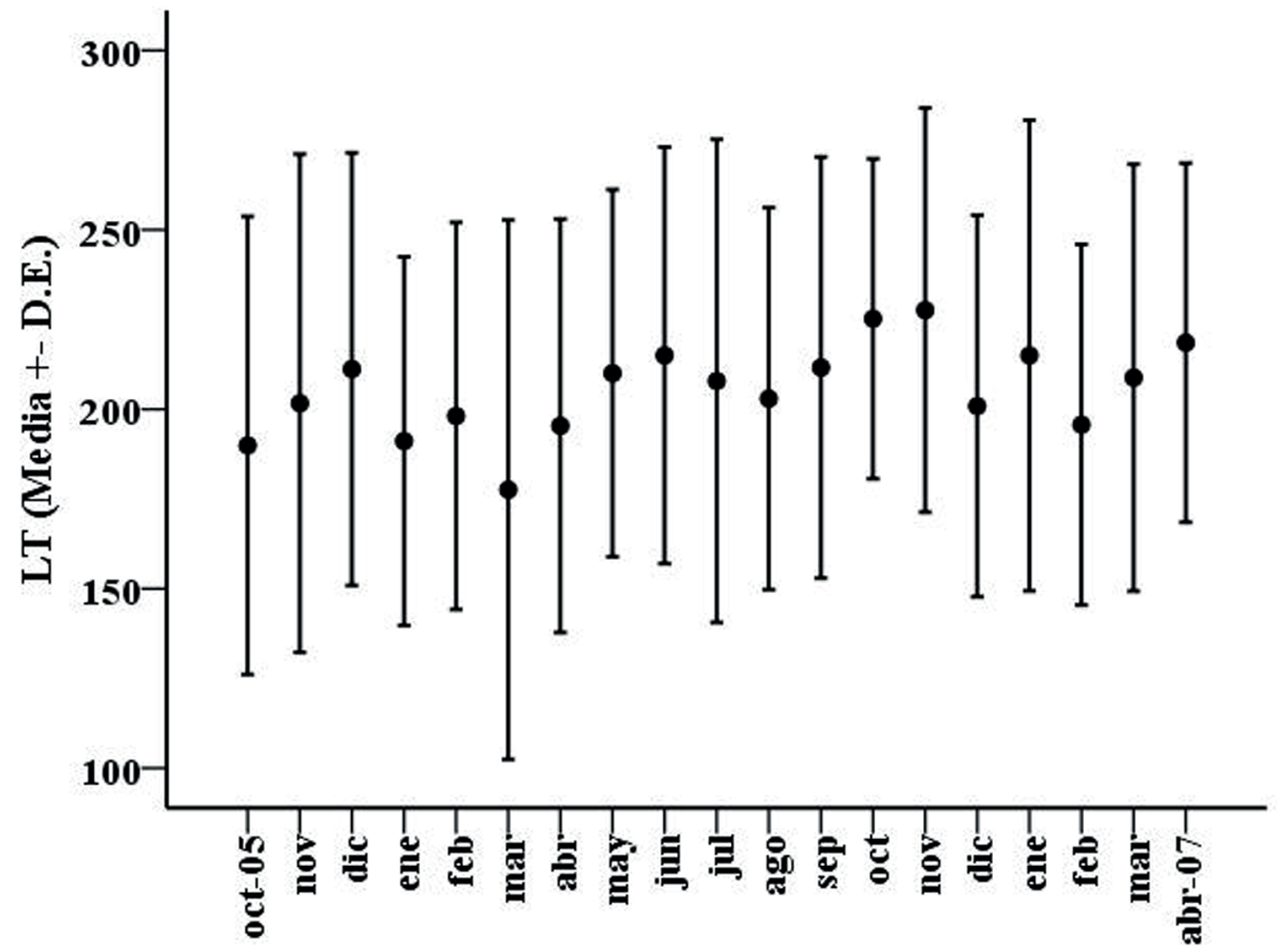

Meses

Fig. 1. Variación mensual de la longitud total (Media \pm D.E.) en Panulirus gracilis capturadas en el Golfo de Montijo, Pacífico panameño entre octubre de 2005 y abril de 2007

Fig. 1. Monthly mean of total length (Mean $\pm \mathrm{SD}$ ) in Panulirus gracilis caught in the Gulf of Montijo, Pacific Panama between October 2005 and April 2007

para las hembras. Si los controles de talla mínima legal se aplican a través de la longitud de cola $(120 \mathrm{~mm})$, el $57.8 \%$ de los machos y el $26.7 \%$ de las hembras estuvieron por debajo del valor (Fig. 2).
Reproducción: La proporción sexual fue favorable para los machos (1.31:1, $\left.\mathrm{X}^{2}=12.2, P<0.001\right)$ (Fig. 3).

La cantidad de hembras maduras fue notoria durante todo el periodo de muestreo, con picos marcados 

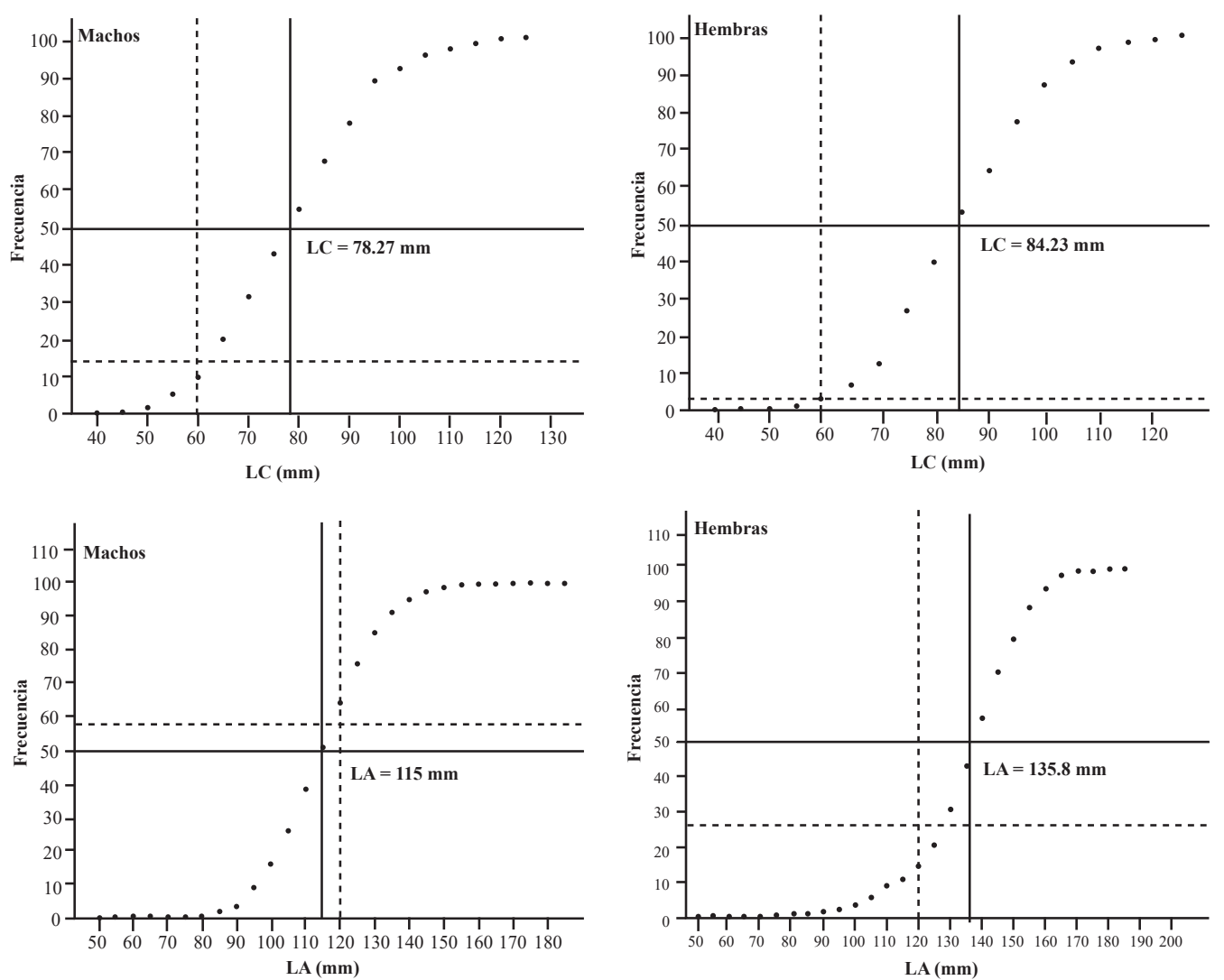

Fig. 2. Frecuencia acumulada de la longitud del cefalotórax (LC) y de la longitud del abdomen (LA) para hembras y machos de langosta espinosa (Panulirus gracilis) capturadas en el Golfo de Montijo, Pacífico de Panamá. Se indica la talla en la cual se acumula el $50 \%$ de la captura (líneas continuas), para machos y hembras, y la talla legal de captura (líneas punteadas)

Fig. 2. Cumulative frequency of cephalothorax length (LC) and abdominal length (LA) for female and male spiny lobster (Panulirus gracilis) caught in the Gulf of Montijo, Pacific Panama. Continuous lines indicate the size where $50 \%$ of the catch is located for males and females while dotted lines indicate the legal catch size

desde finales de la temporada lluviosa (noviembre) hasta febrero en la temporada seca. Este comportamiento fue común para los años 2005-2006 y 2006 -2007 (Fig. 4).

Se presentaron hembras ovadas durante casi todo el estudio, excepto en febrero y marzo de 2006 y en enero de 2007, meses en los cuales se presentaron hembras con gónadas maduras, pero no ovadas.
La presencia de hembras ovadas fue superior al $50 \%$ entre noviembre de 2005 y enero de 2006 , en mayo, julio y octubre de 2006 y en marzo de 2007. De estos meses, los picos de langostas ovadas se presentaron en noviembre de 2005, julio de 2006 y marzo de 2007 (Fig. 5).

De las 299 hembras analizadas, 114 estaban ovadas $(38 \%)$ y 169 se encontraron maduras $(56 \%)$. De las 


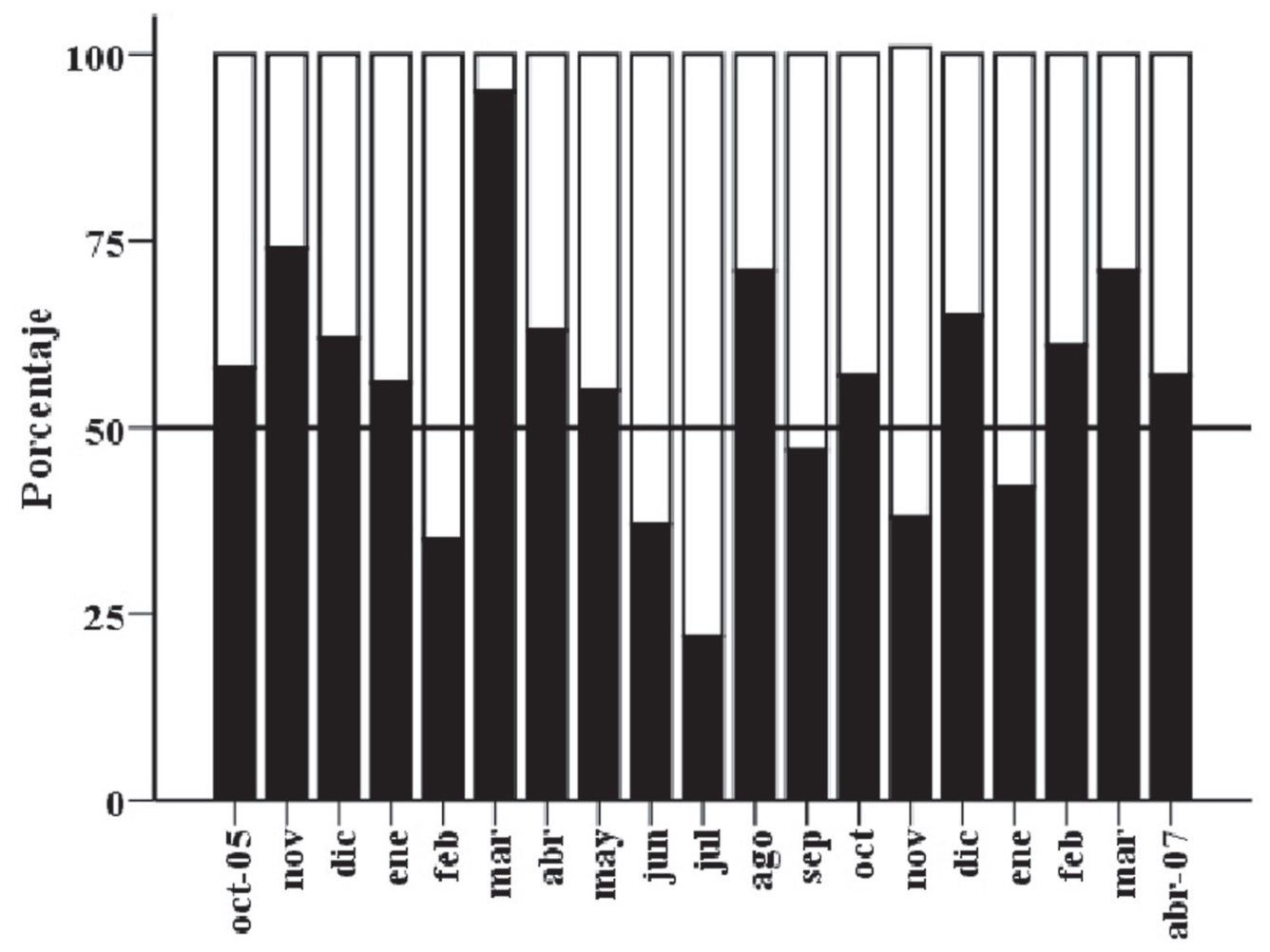

Meses

Fig. 3. Proporción sexual por mes de la langosta espinosa Panulirus gracilis, Golfo de Montijo, Pacífico panameño. Barras oscuras: machos, barras blancas: hembras

Fig. 3. Sex proportion of Panulirus gracilis by month in the Gulf of Montijo, Pacific Panama. Dark bars: male, white bars: female

169 hembras maduras, 60 se presentaron ovadas $(35 \%)$.

\section{Volumen y esfuerzo pesquero:} No existe información sobre volúmenes y esfuerzo de pesca para el GM, pues los pescadores venden su producto a diferentes intermediarios, sin un registro de desembarque. En el PNMGCH, la presencia de un solo intermediario, con registros ordenados de compra entre el 2000 y el 2009, permitió analizar dicha información.

La comercialización de la langosta en el PNMGCH se realiza por peso.
Colas de 2 a 4 oz (langosta chica) y colas de más de $4 \mathrm{oz}$ (langosta grande), pagándose un mejor precio por la langosta de mayor tamaño. También existe la posibilidad de comercializar la langosta entera, chica o grande. En esta clasificación, entre agosto de 2000 y agosto de 2009 se comercializaron como langosta entera grande y chica 35 $377 \mathrm{~kg}$ y $46529 \mathrm{~kg}$, respectivamente; y como cola de langosta grande y chica, 6 $739 \mathrm{~kg}$ y $8974 \mathrm{~kg}$, respectivamente. El 2001 fue el mejor año por la cantidad de langosta comercializada, a partir del cual empezó a caer el volumen de 


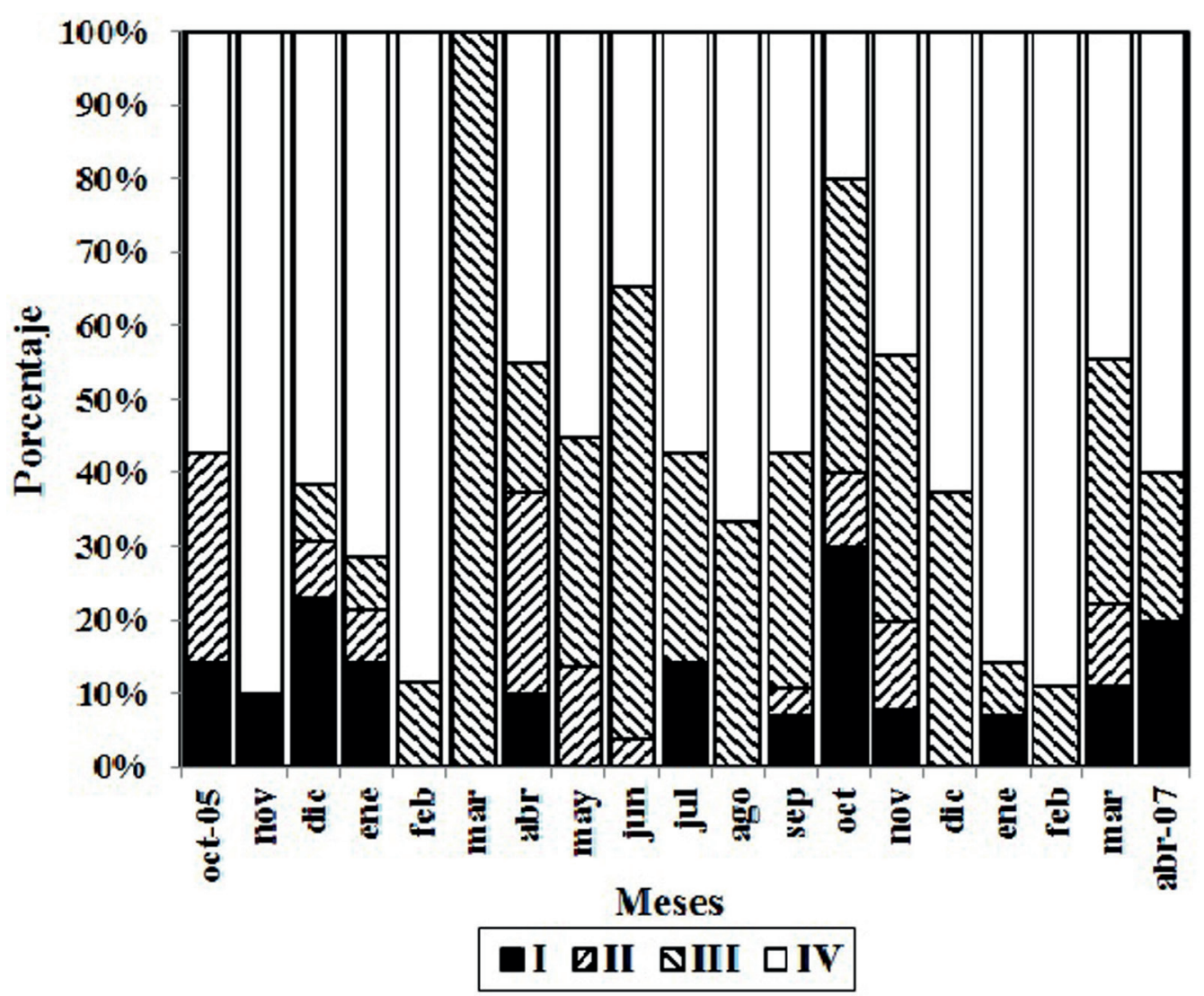

Fig. 4. Porcentajes mensuales de los estadios de desarrollo gonadal en Panulirus gracilis, Golfo de Montijo, Pacífico panameño. I: Reposo. II: Inicio de desarrollo. III. Desarrollo medio. IV: Desarrollo

Fig. 4. Monthly percentages of gonadal stages of Panulirus gracilis. Gulf of Montijo, Pacific Panama. I: Rest, II: Beginning of development, III: Median development, IV: Final or preovulatory development

comercialización, hasta alcanzar los valores más bajos en los años 2005 y 2006, recuperándose ligeramente en el 2007 y el 2008, aunque con valores muy por debajo de los comercializados en los primeros años (Fig. 6).

En todo momento, la langosta clasificada como chica aportó más al total comercializado que la langosta grande. El volumen de cap- tura mostró una correlación positiva y significativa con el esfuerzo pesquero, lo que implicó que las variaciones de los volúmenes de langosta entre el 2000 y el 2009 presentaron un comportamiento similar al esfuerzo pesquero realizado $(P<$ 0.05) (Fig. 6).

La captura por unidad de esfuerzo (CPUE) mostró un descenso sostenido entre el 2000 y el 2003, a 


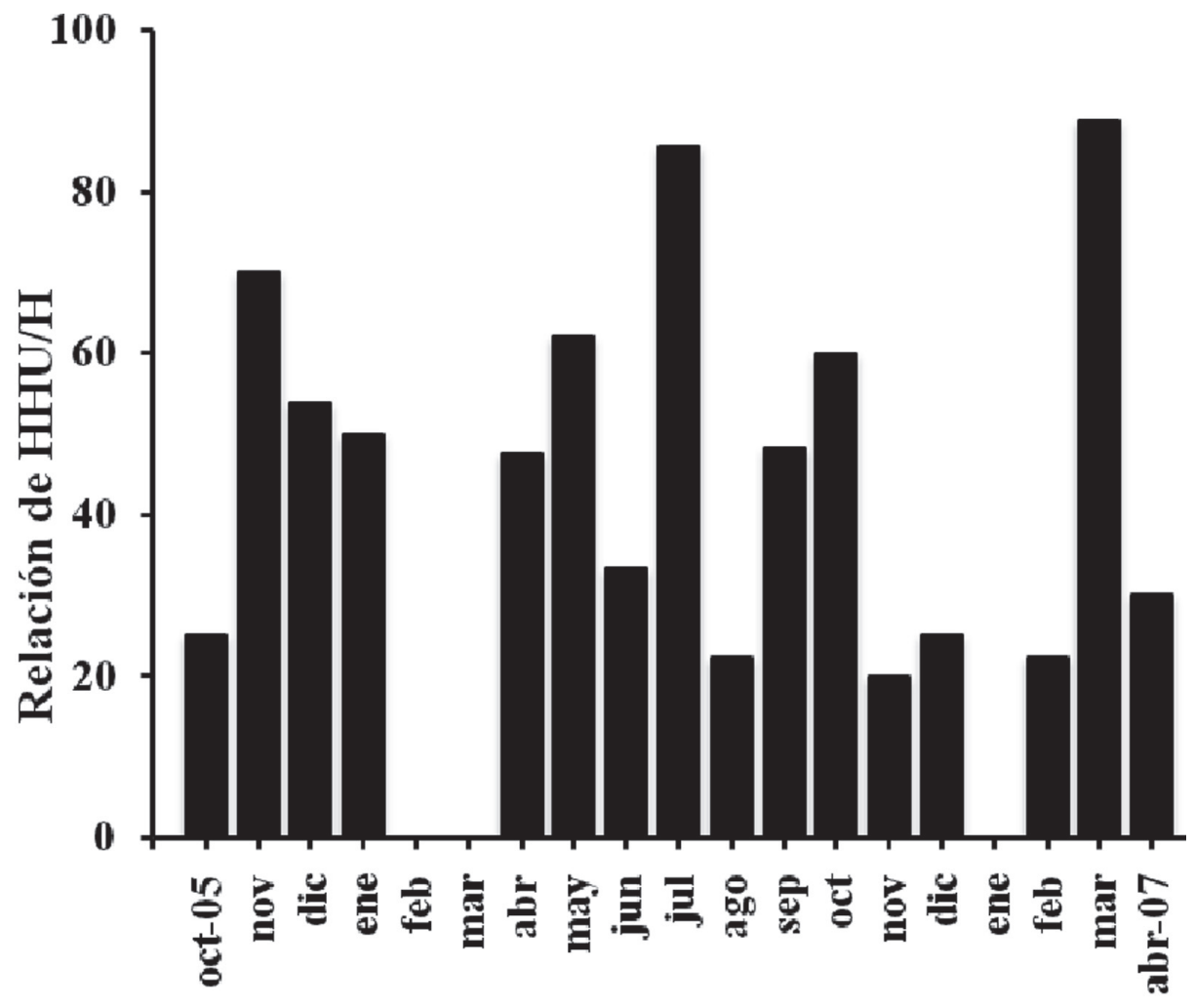

Meses

Fig. 5. Relación entre hembras con huevos (HHU) y hembras (H) en Panulirus gracilis. Golfo de Montijo, Pacífico panameño

Fig. 5. Relationship between females with eggs (HHU) and females $(\mathrm{H})$ in Panulirus gracilis. Gulf of Montijo, Pacific Panama

partir del cual se mantuvo con ligeras oscilaciones hasta el 2006. En el 2007 se recuperó, pero sin alcanzar los valores de los primeros años, manteniendo valores similares hasta el 2009 (Fig. 7).

\section{DISCUSIÓN}

La captura de langosta $P$. gracilis en Panamá se realiza de manera artesanal, con el uso de trasmallos langosteros y por buceo a pulmón, en este último caso se capturan con el uso de chuzos (arpones artesanales) o por capturas manuales. La única normativa vigente para la langosta del Pacífico data de 1981 y establece controles por tallas de cefalotórax y cola, así como por peso de la cola. Durante los muestreos realizados no se observó la implementación de medidas de vigilancia y control, lo cual provoca que algunos pescadores no discriminen entre tallas, sexos 

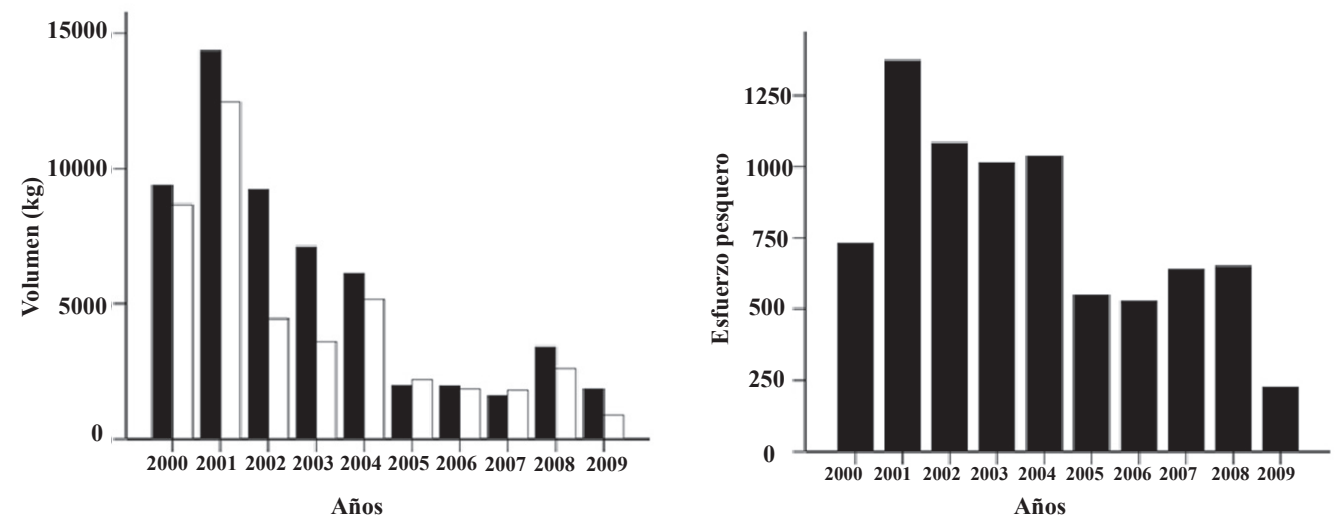

Fig. 6. Volumen de langosta comercializada y esfuerzo pesquero para el Parque Nacional Marino Golfo de Chiriquí. Barras oscuras representan cola de langosta entre 2 y 4 onzas y barras claras representan cola de langosta de más de 4 onzas. Para los años 2000 y 2009 solo se obtuvo información para seis meses

Fig. 6. Commercialized lobster and fishing effort in the Parque Nacional Marino Golfo de Chiriquí. Dark bars represent lobster tails between 2 and 4 ounces and white bars represent lobster tails of more than four ounces. For the years 2000 and 2009 information was obtained only for six months

y condición reproductiva, lo que puede llevar a sobrepescar el recurso (Naranjo-Madrigal, 2011).

Las langostas son capturadas y descabezadas a veces en la propia embarcación de pesca, por lo que a puerto solo llegan las colas. En algunos casos, esta conducta está relacionada con la captura de langosta con chuzos, provocando heridas en el cefalotórax, lo que motiva que se descarte dicha estructura para no dejar la evidencia del uso de arpones. Esto implica que la langosta que se desembarca llega descabezada al punto de compra, donde son pesadas. La ausencia de cefalotórax impide que los controles de talla mínima sean implementados por esta vía, quedando como alternativa la longitud o el peso de la cola.

La no correspondencia entre las tallas mínimas legales establecidas para Panamá, con base en la longitud del cefalotórax $(\mathrm{LC}=60 \mathrm{~mm})$ y la longitud del abdomen (LA = $120 \mathrm{~mm})$, donde se asume que el abdomen es el doble del cefalotórax, se evidenció en los diferentes porcentajes de langostas por debajo de dicha talla, en dependencia de si el espécimen es hembra o macho o si el análisis se realiza con base en la LC o la LA, lo que implica que la normativa vigente no se desarrolló con base en los análisis morfométricos de $P$. gracilis. Este aspecto quedó demostrado al establecer las relaciones entre LC y LA para $P$. gracilis, donde se demostró que una LC de 60 $\mathrm{mm}$ equivale en realidad a una LA de entre 81 y $115 \mathrm{~mm}$ (Guzmán et al. 2008). En este contexto, se recomienda la actualización de la normativa, con base en información 


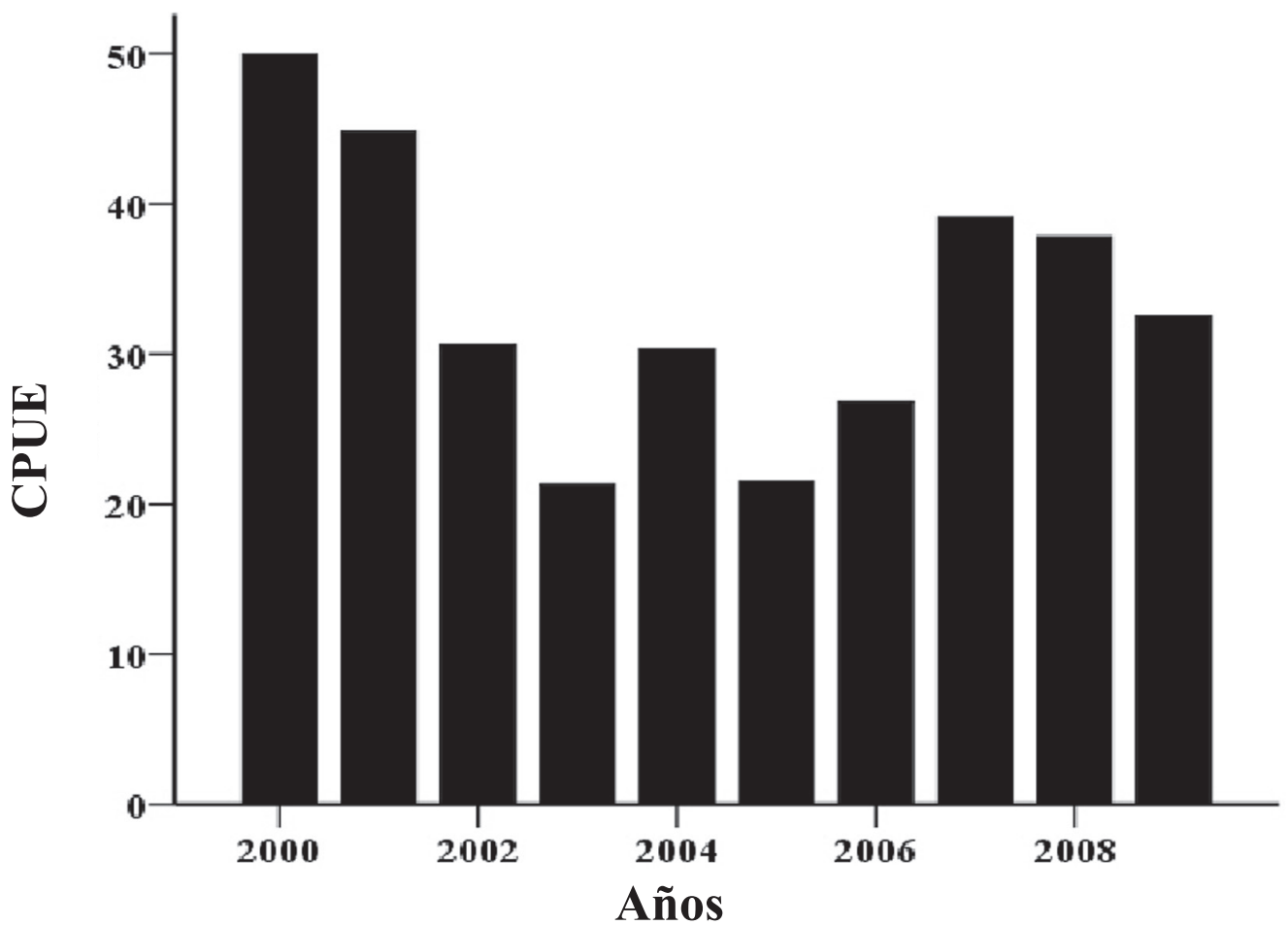

Fig. 7. Captura por unidad de esfuerzo (CPUE) medida como los kilogramos de langosta desembarcados por pescador cada semana

Fig. 7. Catch per unit effort (CPUE) measured as the kilograms of lobster landed by the fisherman every week

generada para esta especie, con un criterio único de control, que por su menor variabilidad y facilidad de manipulación debe ser la LC, por lo que la norma también debe incluir la obligatoriedad de desembarcar los especímenes enteros. Según Guzmán et al. (2008), el cefalotórax presenta la ventaja de ser una estructura rígida y de menor variabilidad y, por lo tanto, es preferible su utilización para medir la longitud de la langosta.

El rango entre las longitudes del cefalotórax de las langostas capturadas en el Golfo de Montijo (LC: 41 a 124 $\mathrm{mm}$ ) es similar a los reportados para otras localidades del Pacífico, donde la especie ha estado sometida a una explotación continua. Para el Pacífico costarricense, Naranjo-Madrigal (2011) reporta LC entre 35.8 y $143.6 \mathrm{~mm}$; y para diferentes localidades del Pacífico mexicano se reportan LC entre 26.1 y 116 mm (Pérez-González et al. 1992), entre 37.1 y 127 mm (Briones-Fourzán \& Lozano-Álvarez, 1992) y entre 50 y $125 \mathrm{~mm}$ (Salazar-Navarro et al. 2006). Sin embargo, la talla promedio de captura, estimada sin discriminar por sexos para LC, es superior para el Golfo de Montijo (81.36 mm), comparada con otras localidades del Pacífico mexicano como en Sinaloa (71.7 mm) y el sur de Baja California 
(76.68 mm, calculada a partir de promedios mensuales entre 1989 y 1990) y el Pacífico de Costa Rica (72.5 mm) (Arzola-González et al. 2011; Pérez-González, 2011; NaranjoMadrigal, 2011). Probablemente, el hecho de que las langostas en el Golfo de Montijo sean más grandes está relacionado con una menor presión pesquera comparada con las restantes localidades, e inclusive en el propio Pacífico panameño, donde tallas promedios de captura de $67 \mathrm{~mm}$ de LC fueron reportadas por Guzmán et al. (2008) para el Archipiélago de las Perlas, Pacífico Oriental de Panamá, donde la actividad extractiva de langosta es más intensa comparada con el Golfo de Montijo, en el que los principales recursos pesqueros son los peces y los camarones penaeidos.

La talla en la que se captura el $50 \%$ de las langostas en el Golfo de Montijo se estimó para la longitud del cefalotórax en $84.8 \mathrm{~mm}( \pm 13.7$, D.E. $)$ para hembras y en $78.9 \mathrm{~mm}( \pm 15.0$, D.E.) para machos. Según Guzmán et al. (2008), la talla media reproductiva para las hembras, estimada para la longitud del cefalotórax, es de $84 \mathrm{~mm}$ y sugieren este valor como el recomendado para la talla legal de captura, que comparado con valores de tallas legales de captura, para otras localidades, son muy similares: Costa Rica $80 \mathrm{~mm}$ de LC (Naranjo-Madrigal, 2011), sur de Sinaloa, México $82.5 \mathrm{~mm}$ de LC (Arzola-González et al. 2011). Para el Golfo de Montijo, implementar los 84 $\mathrm{mm}$ de longitud del cefalotórax como la talla mínima legal de captura, en vez de $60 \mathrm{~mm}$, implicaría que más del 40\% de lo que se captura hoy como legal quedaría por debajo de dicha talla, y en otras localidades, como las Islas de las Perlas, Golfo de Panamá, dicho porcentaje sería superior debido a que las langostas capturadas son de menor tamaño comparadas con el Golfo de Montijo (Guzmán et al. 2008).

Los análisis reproductivos para el GM indican que la langosta espinosa presenta una reproducción continua, con gónadas maduras y hembras ovadas en casi todos los meses del año. Las hembras ovígeras están protegidas por ley, por lo que el establecimiento de controles adecuados garantizaría la reproducción de dichas hembras. Los mayores porcentajes de hembras maduras se presentaron entre noviembre y febrero de 2005 y 2006, por lo que estos meses pueden ser ideales para implementar vedas, en el Pacífico panameño. Si el criterio que se quiere aplicar es la presencia de hembras con huevos visibles, resultaría lógico hacer coincidir la veda del Caribe (1 de marzo al 30 de junio) (OSPESCA, 2009), con la que se implemente para el Pacífico, pues hembras con huevos se presentaron durante este periodo en proporción relativamente alta. De esta manera se evitaría que la presión de pesca se incremente en el Pacífico para suplir la ausencia de pesca en el Caribe.

Para las Galápagos, Ecuador, se registró una disminución de la talla promedio de captura de langostas de $194 \mathrm{~mm}$ a $170 \mathrm{~mm}$ de LC, entre 1997 y el 2004, esto como resultado de la sobrepesca a que ha estado sometido el recurso (Hearn et al. 2005). En el 
caso de Panamá, no se cuenta con información suficiente que permita analizar el comportamiento de la estructura de tallas a lo largo del tiempo, aunque la disminución importante de los volúmenes de comercialización, del esfuerzo pesquero y la CPUE entre los años 2000 y 2009, en la comunidad de Boca Chica, que coincide con la percepción de los pescadores de que las capturas han disminuido, son indicadores de la necesidad de establecer políticas de manejo, que involucre a las comunidades para garantizar la permanencia del recurso y la actividad económica vinculada a su explotación.

\section{AGRADECIMIENTOS}

A la Fundación MarViva, a la Autoridad Nacional del Ambiente (ANAM) y a la Autoridad de los Recursos Acuáticos de Panamá (ARAP) por el apoyo brindando para el desarrollo de esta investigación. A los pescadores del Golfo de Montijo y de Boca Chica por facilitar la manipulación de sus capturas. A los revisores anónimos quienes contribuyeron a mejorar la calidad del artículo.

\section{BIBLIOGRAFÍA}

Arzola-González, J. F., Pérez-González, R., Muñoz-García, I., Gutiérrez-Rubio, Y. \& Flores-Campaña, L. M. (2011). Distribución de tallas de langostas $P a$ nulirus inflatus y $P$. gracilis en la pesquería del sur de Sinaloa, México. Rev. Lat. Am. Rec. Nat., 7(1), 15-20.
Briones, P., Lozano, E., Martínez-Guerrero, A. \& Cortés, S. (1981). Aspectos generales de la biología y pesca de las langostas en Zihuatanejo, Gro., México (Crustacea: Palinuridae). An. Inst. Cienc. del Mar y Limnol. Univ. Nal.Autón. México, 8, 79-102.

Briones-Fourzán, P. \& Lozano-Álvarez, E. (1992). Aspects of the reproduction of Panulirus inflatus (Bouvier) and $P$. gracilis Streets (Decapoda: Palinuridae) from the Pacific coast of Mexico. J. Crustacean Biol., 12(1), 41-50.

Buesa, R. J. \& Mota-Alves, M. I. (1970). Escala de colores para el estudio del ciclo reproductor de la langosta $P$. argus (Latr) en el área del mar Caribe. FAO. Fish. Rep., 71(2), 9-12.

Cámara, A. R., Díaz Olmo, F., Martínez B., J. R., Morón M., M. del C., GómezPonce, C., Tabares, E. \& Vega, A. J. (2004). Directrices de gestión para la conservación y desarrollo integral de un humedal centroamericano: Golfo de Montijo (Litoral del Pacifico de Panamá). Panamá, Panamá: ANAM.

Castillo, A. \& Lessios, H. A. (2001). Lobster fishery by the Kuna Indians in the San Blas Region of Panama (Kuna Yala). Crustaceana, 74(5), 459-475.

Cruz, R. (2002). Manual de métodos de muestreo para la evaluación de las poblaciones de langosta espinosa. Documento Técnico de Pesca. No. 399. Roma, Italia: FAO.

Fischer, W., Krupp, F., Schneider, W., Sommer, C., Carpenter, K. E. \& Niem, V. H. (1995). Guía FAO para la identificación de especies para los fines de la pesca. Pacifico Centro-Oriental. Vol. I. Plantas e invertebrados. Roma, Italia: FAO. 
Guzmán, H. M. \& Tewfik, A. (2004). Population characteristics and cooccurrence of three exploited decapods (Panulirus argus, P. guttatus and Mithrax spinosissimus) in Bocas Del Toro, Panama. J. Shellfish Res., 23, 575-580.

Guzmán, H. M., Cipriani, M., Vega, A. J., López, M. \& Mair, J. M. (2008). Population assessment of the Pacific green spiny lobster Panulirus gracilis in Pacific Panama. J. Shellfish Res., 27(4), 907-915.

Hearn, A., Castrejón, M., Reyes, H., Nicolaides, F., Moreno, J. \& Toral, M. V. (Eds.). (2005). Evaluación de la pesquería de langosta espinosa ( $P a-$ nulirus penicillatus y $P$. gracilis). En Evaluación de las pesquerías en la Reserva Marina de Galápagos (pp. 66-96). Santa Cruz, Galápagos, Ecuador: Fundación Charles Darwin.

Naranjo, H. (2010). Caracterización de los sistemas operacionales, modalidades y artes de pesca utilizados para la captura de la langosta Panulirus gracilis (Streets, 1871) en Guanacaste, Costa Rica. Rev. Mar. Cost., 2, 73-82.

Naranjo-Madrigal, H. (2011). Biología pesquera de la langosta Panulirus gracilis en Playa Lagarto, Guanacaste, Costa Rica. Rev. Biol. Trop., 59(2), 619-633.

OSPESCA, Organización del Sector Pesquero y Acuícola Centroamericano. (2009). Reglamento OSP-02-09 para el ordenamiento regional de la pesquería de la langosta del Caribe (Panulirus argus). Antiguo Cuscatlán, El Salvador: Agencia Española de Cooperación Internacional para el Desarrollo (AECID).

Pérez-González, R. (2011). Catch composition of the spiny lobster Panulirus gracilis (Decapoda: Palinuridae) off the western coast of Mexico. Lat. Am. J. Aquat. Res, 39(2), 225-235.

Pérez-González, R., Flores-Campaña, L. M. \& Núñez-Pastén, A. (1992). Análisis de la distribución de tallas, captura y esfuerzo en la pesquería de langostas Panulirus inflatus (Bouvier, 1895) y $P$. gracilis Streets, 1871 (Decapoda: Palinuridae) en las costas de Sinaloa, México. Proc. San Diego Soc. Natur. Hist., 15, 1-5.

Salazar-Navarro, I., Macías-Sánchez, V., Ramos-González, Á., VirgenÁvila, J. A. \& Esparza-Carvajal, L. E. (2006, octubre). La pesquería de langosta Panulirus inflatus y P. gracilis en las costas de Sinaloa, México. Ponencia presentada en el III Foro Científico de Pesca Ribereña. Jalisco México.

SPSS. (2006). Statistical Package for the Social Sciences for Windows, versión 15.0. Chicago, EE.UU.: SPSS Inc.

Vega, A. J. (2004). Evaluación del recurso pesquero en el Golfo de Montijo. Santiago, Panamá: Impresiones Marín.

Velásquez-Chavarría, L. E. \& GutiérrezGarcía, R. J. (2006). Talla y peso de primera madurez sexual al $50 \%\left(L_{m 50 \%}\right)$ $y\left(P_{m 50 \%}\right)$ para hembras de langosta verde Panulirus gracilis del Pacifico nicaragüense. Managua, Nicaragua: Ministerio de Fomento, Industria y Comercio (MiFIC).

Zar, J. H. (1999). Biostatistical Analysis. New Jersey, EE.UU.: Prentice-Hall. 